\title{
PHENOTYPIC OBSERVATIONS ON MODIFICATION OF POSITION-EFFECT VARIEGATION IN DROSOPHILA MELANOGASTER
}

\author{
J. M. WARGENT and I. J. HARTMANN-GOLDSTEIN \\ Department of Genetics, University of Sheffield, U.K.
}

Received 11.ii.74

\begin{abstract}
Summary
Modification of position-effect variegation by chromosomal rearrangements was studied in single and double inversion strains of Drosophila melanogaster.

When $\operatorname{In}(1) m^{K}$, which causes miniature variegation, and $\operatorname{In}(2 \mathrm{LR}) \operatorname{Rev} v^{B}$, which causes Revolute variegation, were combined in a non-coisogenic background, miniature variegation increased and Revolute variegation decreased.

Introduction of each inversion independently into a coisogenic Amherst background resulted in increased levels of variegation in both strains. When the inversions were combined, the interaction between $m^{K}$ and $R_{e v}{ }^{B}$ was essentially the same as that in the non-coisogenic background.

Revolute variegation was not affected by combining $\operatorname{In}(2 \mathrm{LR}) \operatorname{Rev}^{B}$ with In(1)d1-49, an inversion which does not cause position-effect variegation.

Low culture temperature enhanced variegation; the magnitude of the effect was related to genetic background.

Observations are compatible with the conclusion that interaction between rearranged non-homologous chromosomes modifies variegation only when the rearrangements themselves cause variegation.
\end{abstract}

\section{InTRODUCTION}

A GHANGE in the position of a gene which is brought about by a structural rearrangement having a breakpoint in heterochromatin is commonly associated with the appearance of a variegated phenotype. In Drosophila, the extent to which such position effect is expressed depends on a variety of genetic and environmental factors. These include culture temperature, sex, age, parental constitution, specific modifier genes, Y-chromosome constitution and additional rearrangements in homologous chromosomes (for reviews see Lewis, 1950, and Baker, 1968). The interaction between rearrangements in non-homologous chromosomes has been mentioned in the literature (Morgan et al., 1941; Schultz, 1941; Morgan and Schultz, 1942; but has not been reported in detail and seemed to us to be of potential interest in relation to both variegation and interchromosomal interactions in general. Thus different views on the possible nature of the underlying mechanisms might be formed if combining two rearrangements resulted for example in a mutual increase or decrease of the variegation associated with each.

In the present investigation two inversions, each with one euchromatic and one heterochromatic breakpoint, were combined. One of these inversions was also combined with an inversion having two euchromatic breakpoints. Variegation in single and double inversion strains was observed at two temperatures in the wild type and in a coisogenic background. Several 
techniques were applied to the material. Phenotypic observations are reported in the present paper, cytological and microdensitometric findings have been published or are in preparation.

\section{MAterials AND METHODS}

A homozygous-viable strain $\operatorname{In}(1)$ miniature of Krivshenko, kindly sent to us by Professor J. D. Krivshenko, and a homozygous-lethal strain In(2LR) Revolute of Bridges, obtained from the California Institute of Technology, were used for the induction of variable phenotypes. $\operatorname{In}(1) m^{K}$ was marked with $y^{2} w^{a}$ in the inversion chromosome. Both inversions have one of the breakpoints in heterochromatin and both affect wing phenotype. The wings of $m^{K}$ flies vary in size from wild type to miniature. The wings of $\operatorname{Rev}^{B}$ females heterozygous for the inversion may be curled and held at various angles to the body. In both strains the variable phenotype is due to variegated position effect (Wargent, 1971, 1972). In(1)dl-49, carried in stock In(1)dl-49, y Hw m $\mathrm{g}^{4} / \mathrm{Df}(1) w^{258-11}, y$, obtained from the California Institute of Technology, was used as control rearrangement not associated with position-effect variegation. For details of this and other strains, see Lindsley and Grell (1968).

All phenotypic observations were carried out on structurally heterozygous females. The d1-49 chromosome was combined with a wild type homologue. In the non-coisogenic strains the $\operatorname{Rev}^{B}$ chromosome was balanced with a $v g^{C}$ homologue and in coisogenic strains it was combined with a wild type homologue. To expose the recessive miniature phenotype, the $m^{K}$ chromosome was combined with an $\mathrm{X}$ chromosome bearing the $m$ allele from the stock $m B / m B$ carried in our laboratory. The $m^{K} / m ;+1+$ genotype (hereafter referred to as $m^{K} ;+$ ) was produced by reciprocal crosses between $m^{K} / m^{K} ;+/+$ and $m B / m B ;+/+$. The double inversion strain $\mathrm{dl}-49 /+; \operatorname{Rev}^{B} /+$ (hereafter referred to as $\mathrm{dl}-49 ; \operatorname{Rev}^{B}$ ) was obtained by reciprocal crosses of $\mathrm{d} 1-49 / \mathrm{Df}(1) w^{258-11} ;+/+$ to $+/+; \operatorname{Rev}^{B} / \mathrm{vg}^{C}$. The double inversion strain containing both variegation-inducing rearrangements was synthesised according to a breeding schedule which permitted recognition of the parental source of the $m^{K}$ chromosome, and in which a random parental source of the $\operatorname{Rev}^{B}$ chromosome could be assumed. Strains $m^{K}$ and $R e v^{B}$ were made as coisogenic as possible, the common background being provided by the Amherst wild type which had been inbred in our laboratory by brother-sister mating for more than 20 generations. The $m^{K} / m^{K}$ (Am) strain (referred to as $m^{K}(\mathrm{Am})$ hereafter) was produced by chromosome substitution, since the inversion is phenotypically unmarked, except by association with the recessive mutant alleles $y^{2}$ and $w^{a}$ located distally. The breeding schedule followed is shown in fig. 1. Transmission of the small fourth chromosome was not controlled. The $m B / m B(\mathrm{Am})$ and $R e v^{B} /+(\mathrm{Am})$ strains (hereafter referred to as $m B(\mathrm{Am})$ and $\operatorname{Rev}^{B}(\mathrm{Am})$ respectively) were obtained by repeated backcrosses to Amherst for 14 generations. Calculations based on the formulae for estimating the proportion of unfixed loci after inbreeding (Bartlett and Haldane, 1935) indicate a final Amherst background of 98 per cent in the $m B(\mathrm{Am})$ strain and c. 95 per cent in $\operatorname{Rev}^{B}(\mathrm{Am})$. In the $m^{K}(\mathrm{Am})$ strain the entire $\mathrm{X}$ chromosome was substituted and the Amherst background made up c. 80 per cent.

Observations were carried out on two groups of sibling cultures reared 
at $25^{\circ} \mathrm{C}$. and $14^{\circ} \mathrm{C}$. respectively. All flies were reared on a yeast-glucose medium (Alderson, 1957, modified by Ashburner, 1967) supplemented

\section{Generation}

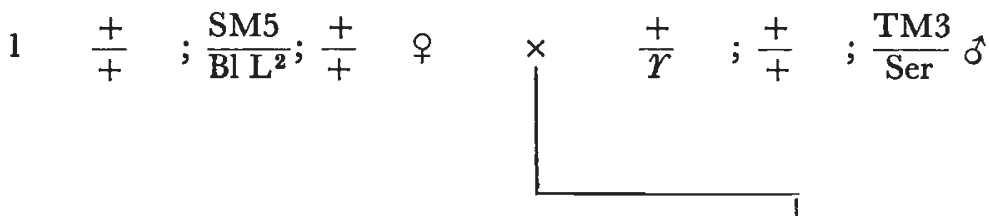

$$
\begin{aligned}
& 2 \quad \frac{\text { FM } 6}{y 1} ; \frac{t}{t} ; \frac{t}{t} \text { ㅇ } \quad \frac{t}{r} ; \frac{\text { SM5 }}{t} ; \frac{\text { TM3 }}{t} \text { o }
\end{aligned}
$$

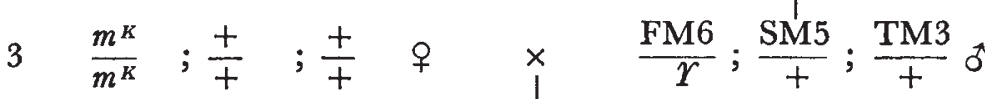

$$
\begin{aligned}
& 4 \quad \frac{\text { FM6 }}{m^{K}} ; \frac{\mathrm{SM} 5}{+} ; \frac{\mathrm{TM} 3}{+} \text { 우 Amherst inbred } \\
& 5 \quad \frac{\text { FM6 }}{\text { inb* }} ; \frac{\text { SM5 }}{\text { inb }} ; \frac{\text { TM3 }}{\text { inb }} \\
& 6 \quad \frac{\text { FM6 }}{\mathrm{inb}} ; \frac{\mathrm{inb}}{\mathrm{inb}} ; \frac{\mathrm{inb}}{\mathrm{inb}}+\quad \times \quad \frac{m^{K}}{r} ; \frac{\mathrm{SM} 5}{\mathrm{inb}} ; \frac{\mathrm{TM} 3}{\mathrm{inb}} \text { } \\
& 7 \frac{m^{K}}{m^{K}} ; \frac{\mathrm{inb}}{\mathrm{inb}} ; \frac{\mathrm{inb}}{\mathrm{inb}}+\quad \text { × } \frac{m^{K}}{r} ; \frac{\mathrm{inb}}{\mathrm{inb}} ; \frac{\mathrm{inb}}{\mathrm{inb}} \sigma
\end{aligned}
$$

* inb refers to the appropriate Amherst chromosome.

$\uparrow$ Males in generation 5 were kept at $18^{\circ} \mathrm{C}$., and subsequently mated to their daughters.

FIG. 1.-Breeding schedule followed for production of $m^{K} / m^{K}$ in Amherst backg round.

with a thin suspension of live yeast for cultures maintained at $14^{\circ} \mathrm{C}$. Parents were bred and aged for 4 days at $25^{\circ}$, transferred to $14^{\circ}$, and eggs were collected for up to 5 days. Adults were transferred back to $25^{\circ}$ and eggs collected for one further day. Eggs which were laid at $25^{\circ}$ were left to complete development at that temperature. Since temperature at the time 
of eclosion can affect the appearance of the wings, the cultures reared at $14^{\circ}$ were transferred to $25^{\circ}$ after the first adult had emerged. Mutant expression was scored on 24-hour-old females which had eclosed at $25^{\circ}$.

Two methods were used for scoring miniature variegation. The frequency of the miniature phenotype was assessed by recording the number of flies whose wings did not project beyond the abdomen, and the frequency of the " crumpled" phenotype occurring in coisogenic $m^{K}$ strains (Wargent, 1971) was assessed by recording the number of such individuals in a population. In non-coisogenic strains the miniature phenotype was also scored by measuring the body weight and wing area. Lightly etherised flies were weighed on a Mettler H20 balance, then killed. The wings were detached, mounted, their outlines recorded on graph paper using a camera lucida, and the mean wing area calculated for each individual. The results were expressed as the ratio of square root of wing area to cube root of body weight.

For scoring Revolute variegation an arbitrary scale ranging from Grade 0 (normal appearance of both wings, i.e. no mutant expression) to Grade 5 was devised. Grades 1-5 represent increasing incidence of mutant expression, taking into account $(a)$ the angle at which the wing is held, $(b)$ extent of curling of the wing and $(c)$ whether one or both wings are affected. For example Grade 5, allocated to maximal mutant expression observed, indicates that both wings are held at an angle of about $90^{\circ}$ and are curled from the base. The mean grade of Revolute expression was calculated by summing the proportion of total flies classified in each grade, each weighted according to the grade expressed. In order to do this we have had to assume that a linear relationship exists between the arbitrarily assigned grades of expression.

\section{Results}

Table 1 shows the effect on Revolute variegation of combining $\operatorname{Rev}^{B}$ and $m^{K}$ in flies reared at different temperatures. At $25^{\circ}$ Revolute expression is similar in single and double inversion strains. At $14^{\circ}$ there is a significant difference in distribution of grades of expression within the single and double inversion populations, a lower proportion of affected individuals showing the more extreme grades in the latter strain. The table also shows that in both strains low temperature increases the number of flies showing Revolute and the proportion of flies showing the higher grades of the Revolute phenotype.

The observation that a difference in variegation is shown by $+; \operatorname{Rev}^{B}$ and $m^{K} ; R_{e v} B$ raises two questions, namely $(a)$ whether a difference in variegation can be shown between $m^{K} ;+$ and $m^{K} ; \operatorname{Rev} v^{B}$, and $(b)$ whether a similar effect can be brought about by combining one of these inversions with a second inversion which is not responsible for a variegated phenotype. To answer the first of these questions, the effect of $\operatorname{Rev}^{B}$ on miniature expression was assessed by comparing the number of flies showing the mutant phenotype in the two strains. The results, presented in table 2, show that miniature variegation in both strains is enhanced by low temperature; at both temperatures, the double inversion strain shows a tendency towards greater variegation than the single inversion strain. Since the results are suggestive but statistically inconclusive, an alternative scoring method was 
devised, based on the proportion of wing area to body weight. In a pilot experiment the method was tested in Amherst wild type and $\operatorname{Rev}^{B}$ and the ratio was found to remain constant at different temperatures. Accordingly, wing area and body weight were measured in $m^{K} ;+$ and $m^{K} ; \operatorname{Rev}{ }^{B}$ strains

TABLE 1

Comparison of Revolute expression in $+; \operatorname{Rev}^{B}$ and $\mathrm{m}^{\mathrm{K}}$; $\operatorname{Rev}^{\mathrm{B}}$ females, expressed as percentage of total number of flies $(\mathrm{n})$ observed

Culture temperature:

$25^{\circ} \mathrm{C}$.

Genotype:

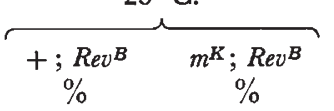

Grade 0

26.9

\section{$22 \cdot 5$}

Grade 1

Grade 2

Grade 3

Grade 4

Grade 5

$23 \cdot 8$

$22 \cdot 5$

$20 \cdot 0 \quad 24 \cdot 0$

$15 \cdot 6$

$20 \cdot 5$

$8 \cdot 8$

$7 \cdot 5$

$5 \cdot 0$

$3 \cdot 0$

Mean Grade

1.7

$1 \cdot 8$

$\underbrace{160}_{\substack{3 \cdot 6 \\ 5}}$

n.s.

\begin{tabular}{|c|c|}
\hline$+\underset{\%}{; \operatorname{Rev} B}$ & $\begin{array}{c}m^{K} ; \operatorname{Rev}{ }^{B} \\
\%\end{array}$ \\
\hline $2 \cdot 7$ & $5 \cdot 7$ \\
\hline 4.9 & $15 \cdot 4$ \\
\hline $15 \cdot 3$ & 30.9 \\
\hline 19.1 & $27 \cdot 9$ \\
\hline $27 \cdot 3$ & $9 \cdot 6$ \\
\hline $30 \cdot 6$ & $10 \cdot 3$ \\
\hline $3 \cdot 6$ & 2.5 \\
\hline 183 & 136 \\
\hline \multicolumn{2}{|c|}{$\begin{array}{l}45 \cdot 8 \\
5 \\
<0 \cdot 001\end{array}$} \\
\hline
\end{tabular}

For explanation of Grades 0-5 and Mean Grade, see Materials and Methods.

reared at $25^{\circ}$ and $14^{\circ}$. Much of the data showed a bimodal frequency distribution. When the data were plotted according to the parental source of the $m^{K}$ chromosome, it became clear that the two modes were the consequence of a parental effect. Results were therefore analysed according to the parental source of the $m^{K}$ inversion and are presented in table 3 .

TABLE 2

Comparison of miniature expression in $\mathrm{m}^{\mathrm{K}}$; + and $\mathrm{m}^{\mathrm{K}}$; $\operatorname{Rev}^{\mathrm{B}}$ females, based on proportion of affected individuals and expressed as percentage of total number of flies ( $\mathrm{n}$ ) observed.

\begin{tabular}{|c|c|c|c|c|}
\hline $\begin{array}{l}\text { Culture } \\
\text { temperature }\end{array}$ & Genotype & $\begin{array}{l}\text { Percentage } \\
\text { miniature }\end{array}$ & $n$ & $x^{2}$ \\
\hline $25^{\circ} \mathrm{C}$. & $\left\{\begin{array}{l}m^{K} ;+ \\
m^{K} ; \operatorname{Rev}^{B}\end{array}\right.$ & $\begin{array}{l}0 \cdot 6 \\
2 \cdot 9\end{array}$ & $\left.\begin{array}{l}170 \\
136\end{array}\right\}$ & $2 \cdot 6$ \\
\hline $14^{\circ} \mathrm{C}$. & $\left\{\begin{array}{l}m^{K} ;+ \\
m^{K} ; \operatorname{Rev} B\end{array}\right.$ & $\begin{array}{l}5 \cdot 8 \\
8 \cdot 6\end{array}$ & $\left.\begin{array}{l}138 \\
152\end{array}\right\}$ & $1 \cdot 1$ \\
\hline
\end{tabular}

The analysis of variance is shown in table 4 . It can be seen that at both temperatures the ratio of wing area to body weight is smaller in the double than in the single inversion strain. The ratio in the single inversion strain is larger at the lower temperature than at the higher; this observation was explained when independent investigation showed that $m^{K}$ strains have a smaller increase in body weight at low temperature than several other strains examined, an effect which is more marked when $m^{K}$ is paternally derived than if it is maternally derived. Because of the unexpected response to temperature, and the ensuing difficulty in interpretation of the ratios obtained for $m^{K}$ strains, this scoring method was not further employed. 
As an approach to the second question posed above, namely whether an effect on variegation results from the presence of a second inversion

\section{TABLE 3}

Comparison of miniature expression in $\mathrm{m}^{\mathrm{K}}$; + and $\mathrm{m}^{\mathrm{K}}$; $\mathrm{Rev}^{\mathrm{B}}$ females, based on relationship of wing area, a $(s q \mathrm{~mm})$ and body weight, $\mathrm{w}(\mathrm{mg})$

\begin{tabular}{|c|c|c|c|c|}
\hline \multirow{2}{*}{$\begin{array}{l}\text { Parental } \\
\text { source of } m^{K} \\
\text { Genotype }\end{array}$} & \multicolumn{2}{|c|}{ Maternal } & \multicolumn{2}{|c|}{ Paternal } \\
\hline & $m^{K} ;+$ & $m^{K} ; R e v^{B}$ & $m^{K} ;+$ & $m^{K} ; \operatorname{Rev} B$ \\
\hline $\begin{array}{l}25^{\circ} \mathrm{C} \\
14^{\circ} \mathrm{C}\end{array}$ & $\begin{array}{l}1.516 \pm 0.0178 \\
1.895 \pm 0.0392\end{array}$ & $\begin{array}{l}1.320 \pm 0.0169 \\
1.895 \pm 0.0717\end{array}$ & $\begin{array}{l}1.470 \pm 0.0181 \\
1.712 \pm 0.0358\end{array}$ & $\begin{array}{l}1 \cdot 361 \pm 0.0340 \\
1.705 \pm 0.0563\end{array}$ \\
\hline $\begin{array}{l}25^{\circ} \mathrm{C} . \\
14^{\circ} \mathrm{C} .\end{array}$ & $\begin{array}{l}1.203 \pm 0.0165 \\
1.373 \pm 0.0348\end{array}$ & $\begin{array}{l}1.043 \pm 0.0185 \\
1.532 \pm 0.0331\end{array}$ & $\begin{array}{l}1.022 \pm 0.0213 \\
1.202 \pm 0.0387\end{array}$ & $\begin{array}{l}1.024 \pm 0.0232 \\
1.486 \pm 0.0332\end{array}$ \\
\hline $\begin{array}{l}25^{\circ} \mathrm{C} . \\
14^{\circ} \mathrm{C} .\end{array}$ & $\begin{array}{l}1 \cdot 158 \pm 0 \cdot 0080 \\
1 \cdot 240 \pm 0 \cdot 0132\end{array}$ & $\begin{array}{l}1 \cdot 134 \pm 0 \cdot 0080 \\
1 \cdot 188 \pm 0 \cdot 0231\end{array}$ & $\begin{array}{l}1 \cdot 206 \pm 0.0123 \\
1 \cdot 235 \pm 0.0174\end{array}$ & $\begin{array}{l}1 \cdot 160 \pm 0 \cdot 0226 \\
1 \cdot 143 \pm 0 \cdot 0198\end{array}$ \\
\hline
\end{tabular}

The ratio was calculated for each fly, and in the table the population mean with its standard error is given. For each population, $n=25$

not associated with position effect, $\operatorname{Rev}^{B}$ was combined with d1-49. The latter is an inversion in chromosome 1 which, like $m^{K}$, is relatively long, but which has two euchromatic breakpoints. The single and double inversion strains $+; R e v^{B}$ and $\mathrm{d} 1-49 ; R_{e v^{B}}$, differ neither in frequency nor in degree of variegation and low temperature was found to enhance variegation in both strains.

TABLE 4

Analyses of variance of ratios presented in Table 3

Parental source of $m^{K}$ :
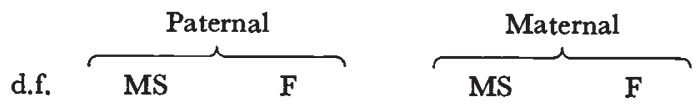

Source of variation:

d.

Between $m^{K} ;+$ and $m^{K} ; \operatorname{Rev} B$ at $25^{\circ} \mathrm{C}$. at $14^{\circ} \mathrm{C}$.

Between $25^{\circ}$ and $14^{\circ}$, within strains

Within temperature/strain

$0.0339-1.4$

$0 \cdot 1160$

Total variation

$\begin{array}{rrr}1 & 0.5262 & 22 \cdot 5 * * \\ 96 & 0.0234 & \end{array}$

$0 \cdot 4749$

99

$$
\mathrm{F}_{(1 / 60)_{0.05}}=4 \cdot 0 .
$$

The tests were carried out on ratios calculated from wing areas in arbitrary units. * and ** denote differences significant at the 5 per cent and 1 per cent levels of probability respectively.

To expose a possible role played by the genetic background in the apparent interaction between $m^{K}$ and $R e v^{B}$, coisogenic single and double inversion strains were compared. From table 5 it is apparent that at the 
lower temperature, which enhances variegation in the single inversion strain only, the double inversion strain shows less extreme Revolute variegation.

TABLE 5

Comparison of Revolute expression in coisogenic $+; \mathrm{Rev}^{\mathrm{B}}$ and $\mathrm{m}^{\mathrm{K}}, \mathrm{Rev}^{\mathrm{B}}$ females, expressed as percentage of total number of files $(\mathrm{n})$ observed

\begin{tabular}{|c|c|c|c|c|}
\hline Culture temperature: & & $5^{\circ} \mathrm{C}$ & & $4^{\circ} \mathrm{C}$ \\
\hline Genotype: & $+\underset{\%}{; \operatorname{Rev}^{B}(\mathrm{Am})}$ & $\underset{\%}{m^{K} ; \operatorname{Rev}^{B}(\mathrm{Am})}$ & $+\underset{\%}{; \operatorname{Rev}^{B}}(\mathrm{Am})$ & $m^{K} ; \operatorname{Rev}_{\%}^{B}(\mathrm{Am})$ \\
\hline $\begin{array}{l}\text { Grade 1 } \\
\text { Grade 2 } \\
\text { Grade 3 } \\
\text { Grade 4 } \\
\text { Grade } 5\end{array}$ & $\begin{array}{l}25 \cdot 5 \\
22 \cdot 4 \\
20 \cdot 6 \\
17 \cdot 6 \\
13 \cdot 9\end{array}$ & $\begin{array}{l}28 \cdot 1 \\
24 \cdot 4 \\
19 \cdot 4 \\
15 \cdot 7 \\
12 \cdot 3\end{array}$ & $\begin{array}{l}11 \cdot 8 \\
16 \cdot 9 \\
19 \cdot 9 \\
23 \cdot 8 \\
27 \cdot 6\end{array}$ & $\begin{array}{l}26 \cdot 1 \\
26 \cdot 6 \\
19 \cdot 7 \\
15 \cdot 4 \\
12 \cdot 3\end{array}$ \\
\hline Mean Grade & $2 \cdot 7$ & $2 \cdot 6$ & $3 \cdot 4$ & $2 \cdot 6$ \\
\hline$n$ & 820 & 867 & 698 & 870 \\
\hline $\begin{array}{l}\chi^{2} \\
\text { d.f. } \\
\mathbf{P}\end{array}$ & & $\begin{array}{l}3.8 \\
4 \\
1.5 .\end{array}$ & & $\begin{array}{l}6 \\
.001\end{array}$ \\
\hline
\end{tabular}

The same type of interaction was observed between strains in the noncoisogenic background (table 1). In the coisogenic strains miniature variegation was scored on the basis of frequency of females showing the mutant phenotypes miniature and crumpled. By this criterion the non-coisogenic double inversion strain showed a trend towards higher incidence of variegation (table 2). Table 6 shows that in the coisogenic double inversion strain there is an increase in the number of miniature females compared with the single inversion strain, which is significant at both temperatures.

TABLE 6

Comparison of miniature expression in coisogenic $\mathrm{m}^{\mathrm{K}}$; + and $\mathrm{m}^{\mathrm{K}}$; $\mathrm{Rev}^{\mathrm{B}}$ females, based on proportion of affected individuals, and expressed as percentage of total number of flies $(\mathrm{n})$ observed.

Culture temperature:

Genotype:

Wild phenotype

Miniature phenotype

"Crumpled" phenotype $25^{\circ} \mathrm{C}$.

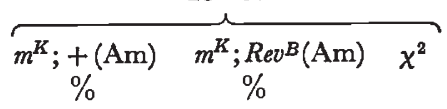

$89 \cdot 6$

$1 \cdot 6$

$8 \cdot 7$

2077

\section{$86 \cdot 2$}

$3 \cdot 2$

$10 \cdot 6$

867
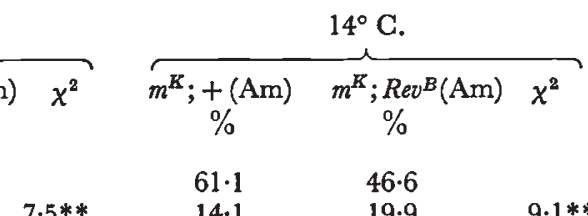

3.8 n.s.

\section{$61 \cdot 1$}

$14 \cdot 1$

$24 \cdot 8$

702
$46 \cdot 6$

$19 \cdot 9$

$33 \cdot 6$

870

** Denotes a difference significant at the 1 per cent level of probability.

The crumpled phenotype, observed only in coisogenic $m^{K}$ strains, also occurs in increased frequency in the double as compared with the single inversion strain, significantly so at $14^{\circ}$. Low culture temperature again enhances variegation in both strains. These results demonstrate that in relation to miniature expression, the behaviour of the non-coisogenic and coisogenic strains differs in degree, not type, of interaction. The effect of 
background on Revolute variegation is demonstrated by comparison of tables 1 and 5: with the exception of the single inversion strain cultured at $14^{\circ}$, the coisogenic strains show more variegation than non-coisogenic strains. A similar background effect operates on miniature variegation: comparison of tables 2 and 6 demonstrates that in coisogenic strains there is an increase in the frequency of miniature flies.

\section{Discussion}

The differences in variegation observed between single and double inversion strains, between strains reared at different temperatures and between strains with different genetic backgrounds show that miniature and Revolute variegation are modified by a number of factors. The effects of these modifiers illustrate the complexity of the phenomenon; it is however possible to draw some conclusions about their interaction and relative effectiveness.

The initial purpose of this work was to determine whether positioneffect variegation is affected by the presence of an additional rearrangement in another chromosome. The evidence for a reduction in the incidence of the Revolute phenotype in the double as compared with the single inversion strains in unequivocal. The behaviour of miniature variegation is not quite as clear cut, since the interpretation of these results is complicated by two factors: the effects of parental source of $m^{K}$ on variegation (currently being investigated) and certain technical limitations in scoring procedures. However, the simplest interpretation of the results is that miniature variegation is enhanced in the double inversion strains. In the coisogenic background, a highly significant increase in the frequency of the mutant phenotypes was observed in the double as compared with the single inversion strains. In the non-coisogenic double inversion strain, there was also an increase in the number of miniature individuals when compared with the single inversion strain, although the increase was not statistically significant. Microscopic examination of wings from $m^{K} / m$ females shows that such wings contain patches of cells of different sizes, in which the smaller cells presumably represent the miniature phenotype (Wargent, 1971). Bearing in mind that the ratio of wing area to body weight remains constant at different temperatures in Amherst wild type and $\operatorname{Rev}^{B}$ (a strain producing wing variegation which is not associated with variation in wing size), the decreased ratio in the double inversion strain variegating for miniature implies increased variegation. Both scoring procedures thus indicate that miniature variegation is increased in the double inversion strain.

The changes in incidence of miniature and Revolute variegation in the double as compared with the single inversion strains could be caused either by the difference in genetic background or by the presence of the additional inversion chromosome. The former possibility seems excluded by the fact that interaction between $\operatorname{Rev}^{B}$ and $m^{K}$ is essentially the same in the wild type and in the Amherst background. Evidently the residual genome cannot be held primarily responsible for the changes in variegation. It is equally evident however, that background plays a role in modifying variegation, although the extent of this effect clearly depends on the genotype and the environment.

One of the questions arising from the observation that $m^{K}$ and $\operatorname{Rev}^{B}$ 
show mutual interaction was whether the presence of an inversion having two euchromatic breakpoints would also affect variegation. If, as we argued above, the changes in variegation in the $m^{K} ; R e v^{B}$ strains result from the presence of the second inversion chromosome, the absence of interaction between $R_{e v}{ }^{B}$ and d1-49 suggests that modification of variegation is related to the presence of an inversion involving a breakpoint in heterochromatin. We cannot exclude the possibility that some other property of the inversion chromosome may be involved. However, we feel our interpretation is the more likely in view of the fact that broken heterochromatin seems implicated in other instances of interchromosomal effects mentioned in the literature (Morgan et al., 1941 ; Schultz, 1941, and pers. comm.; Morgan and Schultz, 1942; Gersh and Ephrussi, 1946; see also Hannah, 1951).

The simplest hypothesis to explain non-homologous chromosome interaction would implicate a diffusible chemical agent, such as might be produced by the specific variegation suppressor loci whose presence has been postulated to explain the effects of the heterochromatic Y chromosome on variegation (Baker and Spofford, 1959; Brosseau, 1964; Hess, 1970). One might visualise that such loci produce, or control production of, an agent capable of acting on any site sensitised by broken heterochromatin. On such an hypothesis the sensitive sites might be expected to compete for the agent. If the agent were a repressor substance, then competition between rearrangements should lead to mutual enhancement of variegation; conversely, the action of an enhancer should lead to mutual suppression of variegation. Our data, and those of Schultz (1941) and Gersh and Ephrussi (1946), strongly indicate that the effect of a particular interaction on the phenotype depends on the rearrangements involved. Such specificity implies a more complex mechanism than the one proposed above. One experimental approach to the problem is through a cytological study of individual cells; the results of such an investigation, to be published elsewhere, suggest that at least two mechanisms affecting chromosomal interactions can be distinguished.

Acknowledgments.-We express our gratitude to Dr B. Burnet for much generous advice and valuable criticism during the course of this work, and to Dr D. J. Goldstein for stimulating discussions and comments. This investigation was supported by a grant (No. 68/1317) from the Science Research Council of Great Britain.

\section{REFERENCES}

Alderson, T. 1957. Culture conditions and mutagenesis in Drosophila melanogaster. Nature, $179,974-975$.

ASHBURNER, M. 1967. Patterns of puffing activity in the salivary gland chromosomes of Drosophila. 1. Autosomal puffing patterns in a laboratory stock of Drosophila melanogaster. Chromosoma (Berlin), 21, 398-428.

BAKER, w. K. 1968. Position-effect variegation. Advances in Genetics, 14, 133-169.

BAKER, W. K., AND SPOFFORD, J. B. 1959. Heterochromatic control of position-effect variegation in Drosophila. Texas University Publication 5914, 135-154.

BARTLETT, M. S., AND HALDANe, J. B. s. 1935. The theory of inbreeding with forced heterozygosis. Fournal of Genetics, 31, 327-340.

BROSsEAU, G. E., JR. 1964. Evidence that heterochromatin does not suppress V-type position effect. Genetics, 50, 237 (Abstract).

GERSH, E. S., AND EPHRussi, B. 1946. The mechanism of position effect-Experiments on the phenotypic expression of position effects in relation to changes in pairing of neighbouring chromosome regions. Proceedings of the National Academy of Sciences, New York, $32,87-94$. 
HANNAH, A. 1951. Localization and function of heterochromatin in Drosophila melanogaster. Advances in Genetics, 4, 87-125.

HESS, O. 1970. Independence between modification of genetic position effects and formation of lampbrush loops by the $Y$ chromosome in Drosophila hydei. Molecular and General Genetics, 107, 224-242.

LEWIS, E. B. 1950. The phenomenon of Position Effect. Advances in Genetics, 3, 73-115.

LINDSLEY, D. L., AND GRELI, E. H. 1968. Genetic variations of Drosophila melanogaster. Carnegie Institute of Washington Publication 627.

MORGAN, T. H., AND SGHULTZ, J. 1942. Investigation on the constitution of the germinal material in relation to heredity. Carnegie Institute of Washington Yearbook, 41, 242-245.

MORGAN, T. H., SCHULTZ, J., AND GURRY, v. 1941. Investigation on the constitution of the germinal material in relation to heredity. Carnegie Institute of Washington Yearbook, 40, 282-287.

schultz, J. 1941. The function of heterochromatin. Proceedings of the 7th International Congress of Genetics (Edinburgh) 1939, 257-262.

wargent, J. M. 1971. Position-effect variegation in wings of Drosophila melanogaster. Drosophila Information Service, 47, 91.

WARGENT, J. M. 1972. Position-effect variegation in the Revolute of Bridges strain of Drosophila melanogaster. Drosophila Information Service, 49, 50-51. 